\title{
DESIGN OF PROBABILISTIC OBSERVERS FOR MASS-BALANCE BASED BIOPROCESS MODELS
}

\author{
Benoît Chachuat and Olivier Bernard
}

\author{
INRIA Comore, BP 93, 06902 Sophia-Antipolis, France \\ fax: +33492387858 \\ email: Olivier.Bernardeinria.fr
}

\begin{abstract}
In this paper, the design of probabilistic observers for mass-balance based bioprocess models is investigated. It is assumed that the probability density of every uncertain parameter, input and/or initial state is known a priori. Then, the probability density of the state variables is obtained, at any time, by considering the image of this initial probability density by the flow of the dynamical system. In comparison to classical open-loop interval observers, the method provides information on the confidence level of the estimates rather than simple upper and lower bounds. Applications to an anaerobic wastewater treatment process are described in order to illustrate the method.
\end{abstract}

Keywords: non-linear estimation, unknown inputs, uncertain parameters, mass-balance models, bioprocesses, anaerobic digestion.

\section{INTRODUCTION}

One of the main difficulties in the monitoring and control of biological reactors lies in the absence, in most applications, of cheap and reliable sensors capable of providing direct, on-line measurements of the biological state variables. The design of state observers, also called software sensors, for the online monitoring of the state variables which are not measurable in real time has thus received increasing attention in the literature, and has given rise to numerous practical applications [Bastin and van Impe, 1995].

Classical observers used in the scope of non-linear systems are, e.g., the extended Kalman filter [Jazwinski, 1974], the extended Luenberger observer [Zeitz, 1987] and, more recently, the high gain observer [Gauthier et al., 1992, Bernard et al., 1998]. But even if these observers are widely used in practice, they assume that the model structure and parameters, and the disturbances affecting the process, are perfectly known. It is well-known that the observed state variables might provide poor estimates of the true values, especially for highly uncertain bioprocesses.
Bioprocess models generally consist of two parts: $(i)$ a part based on mass-balance considerations which requires few phenomenological knowledge; (ii) another part that describes the biological reactions (kinetics) and therefore includes a large part of phenomenological knowledge. Taking advantage of this special structure, it is possible, under certain conditions, to design state observers which do not require the knowledge of the kinetics, by formulating an auxiliary dynamical system [Bastin and Dochain, 1990]. In the counterpart, the resulting observers, called asymptotic observers, are open-loop observers and might be strongly sensitive to biases on the influent masses, e.g., the influent concentrations; in addition, they have weaker convergence properties. More recently, interval observers have been designed in order to deal with these uncertainties [Rapaport and Gouzé, 2003]. The idea is to estimate intervals into which the state is sure to lie, based on guaranteed bounds on the uncertainties such as imprecisions in the influent measurements or model parameters. In this objective, the observation principle is weakened in the sense that the observation error is no longer expected to asymptotically converge to zero. Several applications have been considered for 
biological systems [e.g. Gouzé et al., 2000]. But even if interval observers are more robust than the aforementioned asymptotic observers, poor information is obtained on the estimates since only guaranteed upper and lower bounds are provided on these variables.

In many situations however, more informations than simple upper and lower bounds is available on the uncertainties, e.g. their probability densities. For the uncertain parameters in particular, confidence intervals can be easily obtained from the application of a parameter identification procedure. In this work, we intend to extend the concept of interval observers in order to estimate the probability density of the unmeasured states, at any time, based on the probability densities of the uncertain parameters and state variables at initial time. These observers, called probabilistic observers, are derived here for general mass-balance based models for bioprocesses.

The paper is organised as follow. Some results on both asymptotic and interval observers are first recalled in section 2. Probabilistic observers are considered in section 3, with emphasis on the calculation of the probability densities for the state variable. Finally, an application of probabilistic observers to the estimation of the biomass concentration in an anaerobic digester from real data is detailed to illustrate the method.

\section{ASYMPTOTIC AND INTERVAL OBSERVERS FOR BIOPROCESSES}

A bioprocess operated in a stirred tank bioreactor is often described by means of general mass-balance based models of the following form [Bastin and Dochain, 1990]:

$$
\dot{\boldsymbol{\xi}}=\mathcal{K} \boldsymbol{r}(\boldsymbol{\xi})+D\left(\boldsymbol{\xi}^{i n}-\boldsymbol{\xi}\right)-\boldsymbol{q}(\boldsymbol{\xi})
$$

where $\boldsymbol{\xi} \in \mathbb{R}^{n_{\xi}}$ (resp. $\boldsymbol{\xi}^{i n}$ ) denotes the concentration vector in the liquid phase (resp. in the influent), $\mathcal{K} \in$ $\mathbb{R}^{n_{\xi} \times n_{r}}$ the yield coefficient matrix, $r \in \mathbb{R}^{n_{r}}$ the reaction rate vector, $\boldsymbol{q} \in \mathbb{R}^{n_{\xi}}$ the gaseous exchange vector, and $D \in \mathbb{R}$ the dilution rate.

Throughout the paper, it is assumed that $\left(n_{\xi}-n_{b}\right)$ components of the state variables are measured on-line with $n_{\xi}-n_{b} \geq n_{r}$, and we denote $\boldsymbol{\xi}_{\boldsymbol{a}} \in \mathbb{R}^{n_{\xi}-n_{b}}, \boldsymbol{\xi}_{\boldsymbol{b}} \in$ $\mathbb{R}^{n_{b}}$ the measured and unmeasured state variables respectively. It is also assumed that the gaseous flow rates $\boldsymbol{q}$ are measured on-line. It follows that Eq. (1) can be rephrased as:

$$
\begin{aligned}
& \dot{\boldsymbol{\xi}}_{a}=\mathcal{K}_{a} \boldsymbol{r}(\boldsymbol{\xi})+D\left(\boldsymbol{\xi}_{a}^{i n}-\boldsymbol{\xi}_{a}\right)-\boldsymbol{q}_{a}(\boldsymbol{\xi}) \\
& \dot{\boldsymbol{\xi}}_{b}=\mathcal{K}_{b} \boldsymbol{r}(\boldsymbol{\xi})+D\left(\boldsymbol{\xi}_{b}^{i n}-\boldsymbol{\xi}_{b}\right)-\boldsymbol{q}_{b}(\boldsymbol{\xi})
\end{aligned}
$$

with $\left(\mathcal{K}_{a}, \mathcal{K}_{b}\right),\left(\boldsymbol{\xi}_{a}^{i n}, \boldsymbol{\xi}_{b}^{i n}\right)$ and $\left(\boldsymbol{q}_{a}, \boldsymbol{q}_{b}\right)$ being the induced partition of $\mathcal{K}, \boldsymbol{\xi}^{\text {in }}$ and $\boldsymbol{q}$ respectively.

We state the following hypothesis:
Hypothesis 1. Matrix $\mathcal{K}_{a} \in \mathbb{R}^{\left(n_{\xi}-n_{b}\right) \times n_{r}}$ has full rank.

From hypothesis 1 and a linear change of variables, the following $n_{b}$-dimensional auxiliary system can be derived:

$$
\begin{aligned}
\dot{\boldsymbol{z}} & =-D\left(\boldsymbol{z}-\boldsymbol{z}^{i n}\right)-\mathcal{P} \boldsymbol{q}_{a}-\boldsymbol{q}_{b} \\
\boldsymbol{\xi}_{b} & =\boldsymbol{z}-\mathcal{P} \boldsymbol{\xi}_{a} \\
\text { where: } \quad & \mathcal{P} \triangleq-\mathcal{K}_{b} \mathcal{K}_{a}^{\dagger}
\end{aligned}
$$

and $\mathcal{K}_{a}^{\dagger}$ is a left inverse of $\mathcal{K}_{a}$. Note in particular that, in the auxiliary system $(4,5)$, the measured variable $\boldsymbol{\xi}_{\boldsymbol{a}}$ is used as an input.

The following result holds.

Lemma 1. Under Hypothesis 1 (and provided $D$ is persistently exiting), the solution $\hat{\boldsymbol{\xi}}_{b}$ of the following open-loop observer converges asymptotically towards the solution $\boldsymbol{\xi}_{b}$ of the reduced system (3):

$$
\begin{aligned}
\dot{\hat{\boldsymbol{z}}} & =-D\left(\hat{\boldsymbol{z}}-\boldsymbol{z}^{i n}\right)-\mathcal{P} \boldsymbol{q}_{a}-\boldsymbol{q}_{b} \\
\hat{\boldsymbol{\xi}}_{b} & =\hat{\boldsymbol{z}}-\mathcal{P} \boldsymbol{\xi}_{a}
\end{aligned}
$$

Proof. see, e.g., Bastin and Dochain [1990].

Such observers have however several defects due to their open-loop nature. In particular, it is implicitly assumed that the mass-balance part of the model is perfectly known and that neither the measurements, nor the values of the feeding inputs, nor the estimates of the yield coefficients (matrix $\mathcal{K}$ ) are biased. Otherwise, the predictions of the mass-balance based observer $(6,7)$ will be corrupted and might provide poor estimates of the unmeasured concentrations $\boldsymbol{\xi}_{b}$.

In the case of large uncertainties, it is no longer possible to build exact observers guarantying that the observation errors converges to zero. Therefore, the observation principle must be revisited and the results must be weakened. A complementary approach, called interval observers, provides guaranteed enclosures on the estimated states, whenever upper and lower bounds are known on the uncertain inputs and parameters. Such observers consist in coupling two estimators providing each an over-estimate $\boldsymbol{x}^{+}(t)$ and an under-estimate $\boldsymbol{x}^{-}(t)$ of the unknown state variables $\boldsymbol{x}(t)$ at any time. Details on interval observers can be found, e.g., in Gouzé et al. [2000], Rapaport and Gouzé [2003].

The following proposition applies the concept of interval observers to the auxiliary system (4) derived from the general mass-balance based model (1).

Proposition 1. The following pair of systems is an interval observer for the variables $z(t)$ solution of (4): 


$$
\begin{aligned}
\dot{\boldsymbol{z}}^{+}= & -D \boldsymbol{z}^{+}(t)+D \boldsymbol{z}^{i n+}(t) \\
& -\mathcal{P}^{-}\left(\boldsymbol{p}^{+}, \boldsymbol{p}^{-}\right) \boldsymbol{q}_{a}(t)+\boldsymbol{q}_{b}(t) \\
\dot{\boldsymbol{z}}^{-}= & -D \boldsymbol{z}^{-}(t)+D \boldsymbol{z}^{i n^{-}}(t) \\
& -\mathcal{P}^{+}\left(\boldsymbol{p}^{+}, \boldsymbol{p}^{-}\right) \boldsymbol{q}_{a}(t)+\boldsymbol{q}_{b}(t) \\
\text { with: } \boldsymbol{z}^{+}(0)= & \boldsymbol{\xi}_{b}^{+}(0)+\mathcal{P}^{+}\left(\boldsymbol{p}^{+}, \boldsymbol{p}^{-}\right) \boldsymbol{\xi}_{a}(t) \\
\boldsymbol{z}^{-}(0)= & \boldsymbol{\xi}_{b}^{-}(0)+\mathcal{P}^{-}\left(\boldsymbol{p}^{+}, \boldsymbol{p}^{-}\right) \boldsymbol{\xi}_{a}(t)
\end{aligned}
$$

where the unknown parameters $\boldsymbol{p} \in \mathbb{R}^{n_{p}}$ and inputs $z^{i n} \in \mathbb{R}^{n_{b}}$ are characterised by their upper and lower bounds:

$$
\begin{aligned}
p_{j}^{-} & \leq p_{j} \leq p_{j}^{+}, \forall j=1 \ldots n_{p} \\
z_{i}^{i n^{-}}(t) & \leq z_{i}^{i n}(t) \leq z_{i}^{i n^{+}}(t), \forall t, \forall i=1 \ldots n_{b}
\end{aligned}
$$

and the upper and lower bound matrices $\mathcal{P}^{+}, \mathcal{P}^{-}$are defined componentwise:

$$
\begin{aligned}
& \mathcal{P}_{i, j}^{-}\left(\boldsymbol{p}^{+}, \boldsymbol{p}^{-}\right) \leq \mathcal{P}_{i, j}(\boldsymbol{p}) \leq \mathcal{P}_{i, j}^{+}\left(\boldsymbol{p}^{+}, \boldsymbol{p}^{-}\right) \\
& \forall \boldsymbol{p} \in\left[\boldsymbol{p}^{-}, \boldsymbol{p}^{+}\right], \forall i=1 \ldots n_{b}, j=1 \ldots n_{\xi}-n_{b}
\end{aligned}
$$

\section{Proof. See, e.g., Rapaport and Gouzé [2003].}

Interval observers provide guaranteed bounds on the estimation of the unmeasured variables, given rigorous bounds on the parameters and the inputs. However, the resulting intervals might be large if the uncertainty is high. In this case, it could be interesting to build subintervals on the estimates corresponding to different confidence levels on the uncertainty. Tools to derive such observers are discussed in the next section.

ILLUSTRATIVE EXAMPLE - For the sake of simplicity, we consider a simple model of the anaerobic digestion process by taking into account a single substrate (COD) and single bacterial population, denoted $s$ and $x$ respectively. The biological reaction is represented as:

$$
k s \stackrel{r(\boldsymbol{\xi})=\mu(\cdot) x}{\longrightarrow} x
$$

where $k$ is a yield coefficient, and $\mu(\cdot)$ represents the bacterial growth rate. By assuming perfect mixing in the digester, the simple 2-dimensional dynamical model is obtained:

$$
\begin{aligned}
\dot{x}(t) & =-\alpha D(t) x(t)+\mu(\cdot) x(t) \\
\dot{s}(t) & =-D(t)\left(s(t)-s^{i n}(t)\right)-k \mu(\cdot) x(t)
\end{aligned}
$$

where $\alpha \in[0,1]$ is a coefficient representing the ratio of unattached biomass in the digester and $s^{i n}(t)$ is the concentration of substrate in the feeding stream (it is assumed that no biomass is brought by the influent). In addition, the methane flow rate $q_{\mathrm{CH}_{4}}$ is defined as follow:

$$
q_{\mathrm{CH}_{4}}(t)=k^{\prime} \mu(\cdot) x(t)
$$

with $k^{\prime}$ being the yield coefficient associated with methane production.
Based on model (ADMH), the following asymptotic observer can be derived for the biomass concentration provided that the methane gaseous flow rate is measured on-line:

$$
\dot{\hat{x}}(t)=-\alpha D(t) \hat{x}(t)+\frac{1}{k^{\prime}} q_{\mathrm{CH}_{4}}(t)
$$

This example is in fact a particular case of the asymptotic observer defined in Lemma 1 with $n_{\xi}=n_{b}=1$. Now, if the parameters $\alpha$ and $k^{\prime}$ are uncertain with $\alpha \in\left[\alpha^{-}, \alpha^{+}\right]$and $k^{\prime} \in\left[k^{\prime-}, k^{\prime+}\right]$, an interval observer can be constructed as:

$$
\begin{aligned}
& \dot{\hat{x}}^{-}(t)=-\alpha^{+} D(t) \hat{x}^{-}(t)+\frac{1}{k^{+}} q_{\mathrm{CH}_{4}}(t) \\
& \dot{\hat{x}}^{+}(t)=-\alpha^{-} D(t) \hat{x}^{+}(t)+\frac{1}{{k^{\prime}}^{-}} q_{\mathrm{CH}_{4}}(t)
\end{aligned}
$$

Note that the uncertainty on the measurements can also be handled, e.g., by considering a multiplicative noise $e(t) \in\left[e^{-}, e^{+}\right], \forall t$.

\section{PROBABILISTIC OBSERVERS}

As mentioned before, it is not rare that the influent concentrations and/or model parameters are not known precisely for biological processes. For parameters in particular, estimates as well as confidence intervals can be obtained under the application of a parameter identification procedure. More knowledge than simple upper and lower bounds can obviously be obtained on the unmeasured state variables, e.g. the probability distribution of the estimates.

The construction of interval observers with confidence levels has been previously addressed in Fruchard et al. [2002]. The idea was to approximate the probability densities of the estimates by considering a set of interval observers indexed by the confidence level placed on the uncertain parameters. In this work, we consider a more general approach which consists in estimating the probability density of the state estimates at any time, given known probability densities on the uncertain parameters and states at initial time.

\subsection{Mathematical background}

In mass-balance based bioprocess models of the form (1), the uncertainties may correspond to the yield coefficients in matrix $\mathcal{K}$, to the influent concentrations $\xi^{i n}$, and/or to the unmeasured state variables at initial time $\boldsymbol{\xi}_{b 0}$. In addition, the measurements of the state variables $\boldsymbol{\xi}_{\boldsymbol{a}}$ and the gaseous flow rates $\boldsymbol{q}_{a}, \boldsymbol{q}_{b}$ might be noisy. In order to derive the probability density of the unmeasured state variables $\boldsymbol{\xi}_{b}$, we consider the auxiliary dynamical system $(4,5)$ defined in section 2 . 
Hypothesis 2. The auxiliary dynamical system $(4,5)$ falls into the following class of dynamical systems:

$$
\left\{\begin{aligned}
\dot{\boldsymbol{z}}(t) & =\mathcal{A}(\boldsymbol{p}, t) \boldsymbol{z}(t)+\boldsymbol{b}(\boldsymbol{p}, t) \\
\boldsymbol{\xi}_{\boldsymbol{b}}(t) & =\boldsymbol{z}(t)+\boldsymbol{c}(\boldsymbol{p}, t) \\
\boldsymbol{z}(0) & =\boldsymbol{\xi}_{\boldsymbol{b}}(0)-\boldsymbol{c}(\boldsymbol{p}, 0)
\end{aligned}\right.
$$

where $\boldsymbol{p}=\left(p_{1}, \ldots, p_{n_{p}}\right)^{t} \in \mathbb{R}^{n_{p}}$ denotes the vector of the uncertain parameters, and the components of $\mathcal{A} \in \mathbb{R}^{n_{b} \times n_{b}}, \boldsymbol{b} \in \mathbb{R}^{n_{b}}$ and $\boldsymbol{c} \in \mathbb{R}^{n_{b}}$ are $\mathcal{C}^{1}$ with respect to $p_{1}, \ldots, p_{n_{p}}$. In addition, the uncertain parameters $\boldsymbol{p}$ and initial conditions $z_{0}$ have independent probability densities (p.d.f.).

Remark 1. For sake of simplicity, we consider that parameters $p_{1}, \ldots, p_{n_{p}}$ are constants with known p.d.f..

Notations 1. In the sequel, the following notations are used: $\chi_{0}=\left(\boldsymbol{\xi}_{b 0}^{t}, \boldsymbol{p}^{t}\right), \mathcal{Z}_{0}=\left(\boldsymbol{z}_{0}^{t}, \boldsymbol{p}^{t}\right), \chi_{t}=\left(\boldsymbol{\xi}_{b t}^{t}, \boldsymbol{p}^{t}\right)$ and $\mathcal{Z}_{t}=\left(\boldsymbol{z}_{t}^{t}, \boldsymbol{p}^{t}\right)$. In addition, we denote $f_{\chi_{0}}, f_{\mathcal{Z}_{0}}$, $f_{\chi_{t}}$ and $f_{\mathcal{Z}_{t}}$ their respective p.d.f..

The estimation of the p.d.f. of the unmeasured state variables $\boldsymbol{\xi}_{b}$ at a given time $t$ can be decomposed into three successive steps:

1) estimate the p.d.f. of the random variable $\mathcal{Z}_{0}$ at initial time, from the individual p.d.f. of the unmeasured state variables $\boldsymbol{\xi}_{b 0}$ and the uncertain parameters $\boldsymbol{p}$;

2) compute the image $f_{\mathcal{Z}_{t}}$ at time $t$ of the p.d.f. of $f_{\mathcal{Z}_{0}}$, by the flow of the auxiliary dynamical system (4);

3) estimate the p.d.f. of the unmeasured state variables $\boldsymbol{\xi}_{b t}$ at time $t$, from the p.d.f. of $\mathcal{Z}_{t}$.

The following Theorem [see, e.g., Grimmett and Stirzaker, 2001] provides a general framework to calculate the image of a given p.d.f. by a $\mathcal{C}^{1}$-diffeomorphism for each aforementioned step.

Theorem 1. Let $\mathcal{U}, \mathcal{V}$ be open subsets of $\mathbb{R}^{n}, K$ be a compact subset of $U$, and $\phi: \mathcal{U} \longmapsto \mathcal{V}$ be a $\mathcal{C}^{1}$ diffeomorphism. If $z$ is a random variable with a p.d.f. $f_{z}$, then the random variable $\omega=\phi(z)$ has a p.d.f. $f_{\omega}$ given by:

$f_{\omega}(\omega)=\left\{\begin{array}{l}f_{z}\left(\phi^{-1}(\omega)\right) \operatorname{det}\left(J_{\phi^{-1}}(\omega)\right) \quad \text { if } \omega \in \phi(K) \\ 0 \quad \text { otherwise }\end{array}\right.$

where $J_{\phi}$ denotes the Jacobian matrix of $\phi$.

For steps 1) and 3), consider the mapping $\psi_{t}$ defined as:

$$
\begin{aligned}
\psi_{t}: \mathcal{U}_{t}^{\prime} \longrightarrow \mathcal{U}_{t} & =\psi_{t}\left(\mathcal{U}_{t}^{\prime}\right) \\
\mathcal{Z}_{t} \longmapsto \chi_{t} & =\left(\boldsymbol{\xi}_{\boldsymbol{b} t}^{t}, \boldsymbol{p}^{t}\right)^{t}
\end{aligned}
$$

where $\mathcal{U}_{t}^{\prime}$ is an open subset of $\mathbb{R}^{n_{b}+n_{p}}$. From Hypothesis 2 , the following property is immediate.
Property 1. $\psi_{t}$ is a $\mathcal{C}^{1}$-diffeomorphism.

For step 2), consider the mapping $\varphi_{0}^{t}$ defined as:

$$
\begin{aligned}
\varphi_{0}^{t}: & \mathcal{U}_{0}^{\prime} \longrightarrow \mathcal{U}_{t}^{\prime}=\varphi_{0}^{t}\left(\mathcal{U}_{0}\right) \\
\mathcal{Z}_{0} \longmapsto \mathcal{Z}_{t} & =\left(\boldsymbol{z}_{t}^{t}, \boldsymbol{p}^{t}\right)^{t}
\end{aligned}
$$

with $\mathcal{U}_{0}^{\prime}$ being an open subset of $\mathbb{R}^{n_{b}+n_{p}}$. The mapping $\varphi_{0}^{t}$ can be explicitly defined. Indeed, from linear systems theory, a general solution of system $(\mathcal{S})$ can be obtained from:

$\boldsymbol{z}_{t}=\boldsymbol{z}(t, \boldsymbol{p})=\Phi(0, t, \boldsymbol{p}) \boldsymbol{z}_{0}+\int_{0}^{t} \Phi(\tau, t, \boldsymbol{p}) \boldsymbol{b}(\tau, \boldsymbol{p}) d \tau$

where $\Phi(\cdot, \cdot, \boldsymbol{p}) \in \mathbb{R}^{n_{b} \times n_{b}}$ is the transition matrix, i.e. the solution of the matrix differential equation:

$$
\begin{aligned}
\frac{d}{d t} \Phi(\tau, t, \boldsymbol{p}) & =\mathcal{A}(t, \boldsymbol{p}) \Phi(\tau, t, \boldsymbol{p}), \forall t \\
\Phi(\tau, \tau, \boldsymbol{p}) & =I_{n_{b} \times n_{b}}
\end{aligned}
$$

Based on the analytical solution of system $(\mathcal{S})$ and Hypothesis 2, it can be shown that the following property holds:

Property 2. $\varphi_{0}^{t}$ is a $\mathcal{C}^{1}$-diffeomorphism.

Proposition 2. The p.d.f. $f_{\chi_{t}}$ corresponding to the image $\chi_{t}=\left(\boldsymbol{\xi}_{\boldsymbol{b}_{t}}^{t}, p^{t}\right)^{t}$ of the random variable $\chi_{0} \in$ $K_{0} \subset \mathcal{U}_{0}$ is given by:

$$
f_{\chi_{t}}\left(\chi_{t}\right)=\left\{\begin{array}{l}
\left.\frac{f_{\chi_{0}}\left(\psi_{0} \circ \varphi_{0}^{t}-1\right.}{\operatorname{det} \Phi(0, t, \boldsymbol{p})} \psi_{t}^{-1}\left(\chi_{t}\right)\right) \\
\quad \text { if } \quad \chi_{t} \in K_{t}=\varphi_{0}^{t}\left(K_{0}\right) \\
0 \quad \text { otherwise }
\end{array}\right.
$$

Proof. From Properties 1 and 2, the transformations in steps 1), 2) and 3) are $\mathcal{C}^{1}$-diffeomorphisms. The overall transformation $\psi_{0} \circ \varphi_{0}^{t-1} \circ \psi_{t}^{-1}$ is therefore a $\mathcal{C}^{1}$-diffeomorphism (chain rule of differentiation), and Theorem 1 applies. Since,

$$
\operatorname{det} J_{\psi_{0}}=\operatorname{det} J_{\psi_{t}}=I_{n_{b}+n_{p}}
$$

then,

$$
\operatorname{det} J_{\left(\psi_{0} \circ \varphi_{0}^{t-1} \circ \psi_{t}^{-1}\right)}=\operatorname{det} J_{\varphi_{0}^{t-1}}
$$

In addition,

$\operatorname{det} J_{\varphi_{0}^{t}}=\operatorname{det}\left(\begin{array}{cc}\Phi(0, t, \boldsymbol{p}) & \star \\ 0_{n_{p} \times n_{b}} & I_{n_{p} \times n_{p}}\end{array}\right)=\operatorname{det} \Phi(0, t, \boldsymbol{p})$ which completes the proof.

Finally, individual p.d.f. for the unmeasured state variables $\boldsymbol{\xi}_{b}$ can be obtained, at any time $t$, by integrating the joint state/parameter p.d.f. with respect to the uncertain parameters $\boldsymbol{p}$. 
ILLUSTRATIVE EXAMPLE (continued) - A probabilistic observer can be derived for the biomass concentration from auxiliary system (8). In this case, note that $\psi_{t}=\psi_{0}^{-1}=I$. Moreover,

$$
\begin{array}{r}
\hat{x}(t)=\Phi(0, t, \alpha) \hat{x}_{0}+\frac{1}{k^{\prime}} \int_{0}^{t} \Phi(\tau, t, \alpha) q_{\mathrm{CH}_{4}}(\tau) d \tau \\
\text { where: } \Phi(\tau, t, \alpha)=\exp \left[-\alpha \int_{\tau}^{t} D(s) d s\right]
\end{array}
$$

and

$$
\operatorname{det}\left(J_{\varphi_{0}^{t}}\right)=\exp \left[-\alpha \int_{0}^{t} D(\tau) d \tau\right]
$$

\subsection{Application to the anaerobic digestion process}

The illustrative example presented throughout the paper is continued hereafter.

Experimental data from a pilot scale digester located at the LBE-INRA in Narbonne (France) are considered. This digester consists of a $1 \mathrm{~m}^{3}$ UASB reactor and is fed with industrial wine distillery vinasses obtained from local wineries. Both dilution rate $D$ and methane molar outflow rate $q_{\mathrm{CH}_{4}}$ are available from on-line measurements over a 40-days period. More details on the plant configuration and the experimental data can be found, e.g., in Bernard et al. [2001].

The objective is to design a probabilistic observer estimating the biomass concentration $x$ in the digester based on the auxiliary system (8). The uncertainty corresponds to the yield coefficient $k^{\prime}$, the parameter $\alpha$ and the concentration of biomass $\hat{x}_{0}$ at initial time.

- The value of $\frac{1}{k^{\prime}}$ was estimated off-line based on mass-balance considerations, along with its standard deviation:

$$
\frac{1}{k^{\prime}}=6.2410^{-3} \pm 2.1 \%
$$

It is assumed that the probability density for this parameter is Gaussian. In addition, the following 100\%-confidence interval was obtained from statistical considerations, $\left[{\frac{1}{k^{\prime}}}^{-} ;{\frac{1}{k^{\prime}}}^{+}\right]=$ $\left[4.710^{-3} ; 7.810^{-3}\right]$.

- Parameter $\alpha$ is much more uncertain, and is therefore attributed a uniform probability distribution between bounds $\left[\alpha^{-} ; \alpha^{+}\right]=[0.3 ; 0.7]$.

- Finally, the following conservative bounds are considered for the biomass concentration at initial time, $\left[\hat{x}_{0}^{-} ; \hat{x}_{0}^{+}\right]=\left[0 ; 5 \mathrm{mg} \cdot \mathrm{L}^{-1}\right]$.

Also note that the experimental data have been filtered and, for the sake of simplicity, no measurement errors are taken into account here.

The results obtained from the application of the probabilistic observer are pictured in Fig. 1. The probability density of the biomass concentration are plotted every 4 days (full line), along with both upper and lower bounds on the estimates (dotted line). Experimental points resulting from the off-line measurement of the VSS concentration are also plotted in order to validate the results (errorbars on the measurements are considered since the VSS and biomass concentrations are linked as $\operatorname{VSS}=\alpha \times x$ ).

Roughly speaking, it can be seen that the $100 \%$ confidence bounds (i.e., the bound corresponding to the interval observer) correctly enclose the experimental measurements. Note that the two data points strongly differing from the others at time $t=17$ day correspond to a large increase in the influent flow rate leading to a situation not described by the simple $(\mathrm{ADMH})$ model. Also note that the bounds are rather conservative since they correspond to intervals of about $3 \mathrm{mg} . \mathrm{L}^{-1}$.

The probability density of $\hat{x}$, which is uniform at initial time by assumption, quickly changes during the first 15 days, and then reaches a pattern that remains roughly similar until final time. Insightful information can be deduced from these results:

- The bounds on $\hat{x}$ can be significantly improved with respect to the $100 \%$-confidence bounds since a significant part of the range has a null probability.

- The maximum likelihood estimate is close to the lower bound during all the operation.

A straightforward interpretation can be obtained from the resulting p.d.f. by extracting the mean and standard deviation values of $\hat{x}$. These values are pictured in Fig. 2. It can be seen in particular that the estimates are close to the experimental measurements, and the interval corresponding to the standard deviation of $\hat{x}$ mainly encloses these measurements.

\section{CONCLUSIONS}

In this paper, the design of probabilistic observers was investigated for general mass-balance based bioprocess models. It was shown how the probability density of the unmeasured state variables can be derived from the knowledge of the probability density of the uncertain parameters and/or measurement noises. An illustration of the method for a simple anaerobic digestion model was then presented. Applications to higher dimensional dynamical are currently being investigated.

Although only an asymptotic rate of convergence is guaranteed in this paper, it worth pointing out that this rate can be chosen in some cases [Rapaport and Gouzé, 2003]. Such probabilistic observers can therefore be used as tools to improve bioprocess monitoring. In addition, they could also be applied in the scope of bioprocess supervision and fault detection, e.g., by defining a given tolerance expressed in term of a threshold confidence level. 


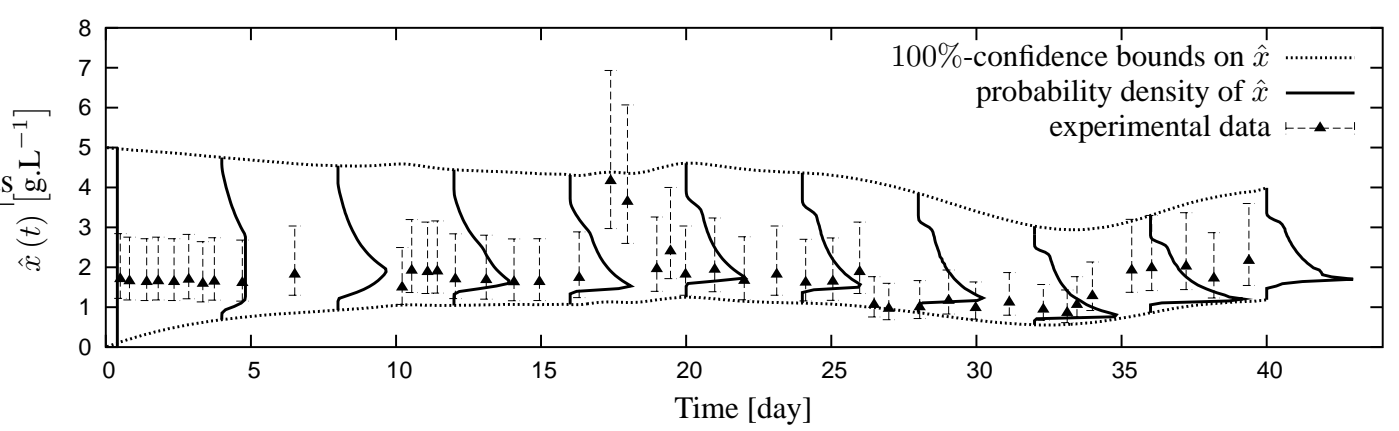

Figure 1. Probability density of the estimated biomass concentration in the digester.

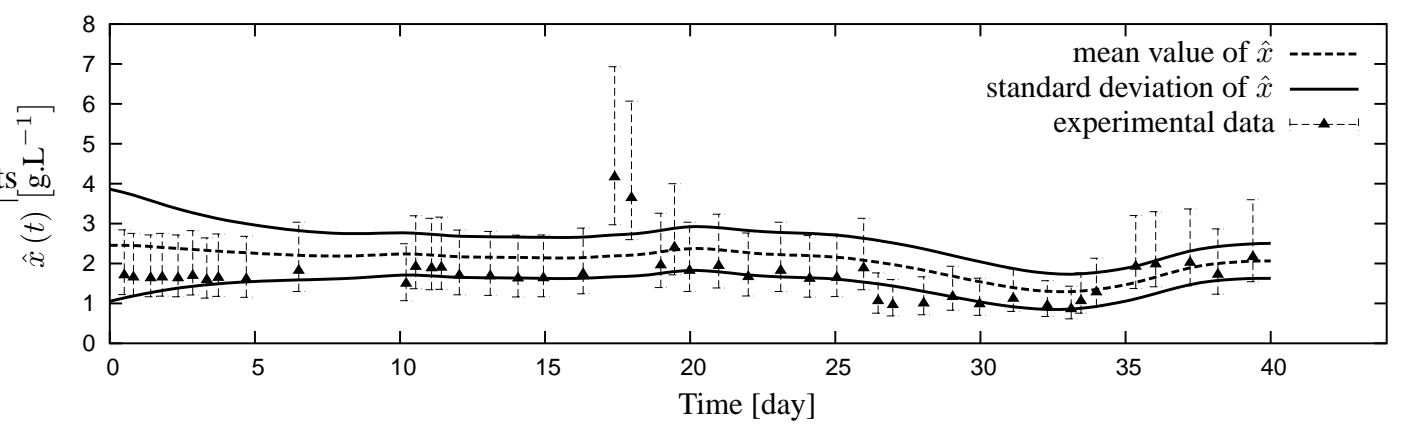

Figure 2. Mean value and standard deviation of the estimated biomass concentration in the digester.

Acknowledgments: This work has been carried out with the support provided by the European Commission, Information Society Technologies program, Key action I Systems \& Services for the Citizen, contract TELEMAC number IST-2000-28256. This information is provided under the sole responsability of the author and does not necessarily represent the opinion of the European Commission, which is not responsible for any use that might be made of it.

\section{REFERENCES}

G. Bastin and D. Dochain. On-line estimation and adaptive control of bioreactors. Elsevier, Amsterdam, The Netherlands, 1990.

G. Bastin and J. van Impe. Nonlinear and adaptive control in biotechnology: a tutorial. European Journal of Control, 1(1):1-37, 1995.

O. Bernard, Z. Hadj-Sadok, D. Dochain, A. Genovesi, and J.-P. Steyer. Dynamical model development and parameter identification for an anaerobic wastewater treatment process. Biotechnol. Bioeng., 75(4): 424-438, 2001.

O. Bernard, G. Sallet, and A. Sciandra. Nonlinear observers for a class of biological systems. Application to validation of a phytoplanktonic growth model. IEEE Trans. Automat. Control., 43:1056$1065,1998$.

M. Fruchard, O. Bernard, and J.-L. Gouzé. Interval observers with confidence levels. Application to the activated sludge process. In Proc. 15th IFAC World Congress, 21st-26th July, 2002, Barcelona,Spain, 2002.

J.-P. Gauthier, H. Hammouri, and F. Celle. A simple observer for nonlinear systems - Applications to bioreactors. IEEE Trans. Automat. Control., 37: 875-880, 1992.

J.-L. Gouzé, A. Rapaport, and M. Z. Hadj-Sadok. Interval observers for uncertain biological systems. Ecological Modelling, 133:45-56, 2000.

G. R. Grimmett and D.R. Stirzaker. Probability and Random Processes. Oxford University Press Inc., New York, 3rd edition edition, 2001.

A. H. Jazwinski. Stochastic processes and filtering theory. Academic Press, New York, 1974.

A. Rapaport and J.-L. Gouzé. Parallelotopic and practical observers for non-linear uncertain systems. International Journal of Control, 76(3):237-251, 2003.

M. Zeitz. The extended Luenberger observer for nonlinear systems. System \& Control Letters, 9: 149-156, 1987. 